\title{
Innovation in Children's Anticoagulant Drugs
}

\author{
Zhoupeng $\mathrm{Wu}^{1}$ and Yukui $\mathrm{Ma}^{1, *}$ \\ ${ }^{1}$ Department of Vascular Surgery, West China Hospital, Chengdu, China \\ "Corresponding author: Department of Vascular Surgery, West China Hospital, 37 GuoXue Alley, Chengdu, China. Email: hxyymyk@126.com
}

Received 2020 February 27; Revised 2020 March 31; Accepted 2020 April 06.

Keywords: Thrombosis, Anticoagulant, Pediatric

\section{Background}

The incidence of venous thromboembolism (VTE) in children has been steadily increasing over the past 20 years, mainly due to increased use of central venous access devices and significant advances in child management under chronic and/or severe medical conditions (1). The rapid growth of VTE in pediatric tertiary care hospitals across the United States has become a problem that cannot be ignored. Pediatricians must deal with the fact that most of the latest treatment recommendations for VTE in children are derived from adult-related research in the treatment of VTE (2). Understanding the reasons for the rapid increase in children with this disease, conducting identification of such children and research on optimal treatment strategies is crucial.

Firstly, the coagulation system of the youngest child (< 1 year old) is not yet fully mature, so the level of anticoagulant action is much lower in children of this age group, and these children need to be treated due to differences in pharmacokinetics in drug metabolism.

Secondly, children who require anticoagulant therapy often have severe underlying conditions that may exacerbate the risk of severe bleeding or severe adverse drug reactions, making the use of anticoagulants more challenging.

Finally, there are relatively few data from prospective clinical trials of anticoagulant therapy in children compared to adults, making anticoagulation decisions more difficult.

Standard anticoagulants currently widely used in children include unfractionated heparin, low molecular weight heparin and VKA (Vitamin K Antagonists) (mainly warfarin). These drugs are multi-target drugs because they exert their effects through anticoagulant mechanisms against a variety of coagulation proteins. These drugs have been used in children's anticoagulant therapy for decades, and despite the lack of prospective studies, they still have the trust of a large number of pediatricians. Due to the relative lack of alternatives, experienced pediatricians can only use these drugs, making them the most commonly used anticoagulants to date. However, looking ahead, the prospects for children's anticoagulant drugs will change dramatically, conventional drugs will be largely replaced by alternative drugs, and in the future, new oral anticoagulants will be used prospectively on a global scale. Fortunately, three studies on anticoagulants in children were officially released in 2019.

\section{New Studies}

\subsection{EINSTEIN-Jr Phase III}

EINSTEIN-Jr Phase III (3) is a randomized, open-label study comparing the efficacy and safety of 20 mg Rivaroxaban with the equivalent dose in a standard anticoagulation regimen for children with any type of acute VTE. The study runs for 4 years and is expected to include 500 children. In the study, patients received open-label, weightadjusted doses of Rivaroxaban $(n=335)$ or standard anticoagulant $(n=165)$ therapy in a $2: 1$ ratio. It is worth mentioning that this study is the largest pediatric study ever to treat VTE and the first study to evaluate a direct oral anticoagulant for this population. In the recurrent VTE and major bleeding composite endpoint (net clinical benefit), the incidence was $1.2 \%$ in the Rivaroxaban group and $4.2 \%$ in the standard anticoagulation group. Imaging tests were performed at baseline and at the end of the treatment period. The reduction in thrombus burden further confirmed the efficacy of Rivaroxaban: the initial elimination rate of VTE was $38.5 \%$ in the Rivaroxaban group and $26.1 \%$ in the standard anticoagulation group.

\subsection{DIVERSITYTrial}

The DIVERSITY trial (4) is a randomized, open-label, parallel-grouped, non-inferiority study involving approximately 180 VTE patients, aged 0 to 18 years, who initially re- 
ceived UFH (unfractionated heparin) or LMWH (low molecular weight heparin) treatment and are expected to require $\geq 3$ months of anticoagulant therapy. Initial doses will be calculated using a proposed dosing algorithm. The composite effect endpoint was VTE recurrence, VTE-related death, and thrombolysis rate. Secondary endpoints include safety (rate of bleeding events) and pharmacokinetic/pharmacodynamic relationship. The results of the DIVERSITY trial showed that Dabigatran was not inferior in treating high-risk children with VTE compared with standard therapy, and the incidence of bleeding was similar.

\section{Secondary VTE Prevention Study of Dabigatran}

This open-ended, single-arm, prospective cohort study, a phase III trial is the first study to target direct oral anticoagulants in children to prevent extended VTE treatment (5). In this study, approximately 200 children were enrolled who were treated with Dabigatran for 12 months. The primary endpoints of the study included VTE recurrence, bleeding events, and 6-month and 12-month mortality. The results of the study showed that the overall incidence of VTE recurrence and major bleeding events were lower. Compared to standard therapies, Dabigatran has "good safety results" for children with VTE or for those who have a persistent risk of thrombosis.

\section{Conclusions}

Over the years, the diagnosis and incidence of VTE has risen sharply, and the incidence of VTE in children is very high. Although we currently have treatments for VTE, pediatric patients still need effective, safer and more convenient treatment options. Therefore, we aimed to investigate whether the proven safety and efficacy of novel oral anticoagulants in adult VTE patients are equally applicable to pediatric patients. Encouragingly, these studies show that they have similar safety and efficacy in the treatment and prevention of VTE in children. Standard anticoagulants used in children, namely unfractionated heparin, LMWH and VKA, have significant limitations. Prospective studies of novel oral anticoagulants have established dose, safety and efficacy, indications, and may change the norms and patterns of anticoagulation in children in the future, and even change the guidelines for anticoagulants in this age group. Selective prescribing of anticoagulants combined with other medications and the promotion of health behavior, change form towards a comprehensive approach to treatment.

\section{Footnotes}

Authors' Contribution: Drafting of the manuscript: Zhoupeng $\mathrm{Wu}$; critical revision of the manuscript for im- portant intellectual content: Yukui Ma.

Conflict of Interests: There were no conflict of interest and legal liability in our report.

Funding/Support: There was no funding/support.

\section{References}

1. Jumani K, Advani S, Reich NG, Gosey L, Milstone AM. Risk factors for peripherally inserted central venous catheter complications in children. JAMA Pediatr. 2013;167(5):429-35. doi: 10.1001/jamapediatrics.2013.775. [PubMed: 23549677]. [PubMed Central: PMC3647026].

2. Monagle P, Cuello CA, Augustine C, Bonduel M, Brandao LR, Capman T, et al. American Society of Hematology 2018 Guidelines for management of venous thromboembolism: treatment of pediatric venous thromboembolism. Blood Adv. 2018;2(22):3292-316. doi: 10.1182/bloodadvances.2018024786. [PubMed: 30482766]. [PubMed Central: PMC6258911].

3. Lensing AWA, Male C, Young G, Kubitza D, Kenet G, Patricia Massicotte M, et al. Rivaroxaban versus standard anticoagulation for acute venous thromboembolism in childhood. Design of the EINSTEIN-Jr phase III study. Thromb J. 2018;16:34. doi: 10.1186/s12959-018-0188-y. [PubMed: 30598642]. [PubMed Central: PMC6302520].

4. Albisetti M, Brandão L, Bomgaars L. Efficacy and safety of dabigatran etexilate for treatment of venous thromboembolism in paediatric patients-results of the DIVERSITY trial. Res Pract Thromb Haemost. 2019;3(Suppl 1):139-40.

5. Brandão LR; Albisetti M; Halton J; Bomgaars L; Chalmers E; Mitchell LG, et al. Safety of Dabigatran Etexilate for Secondary Prevention of Venous Thromboembolism in Paediatric Patient. Res Pract Thromb Haemost. 2019;3(Suppl 1):138-9. 